\title{
Modal Analysis for the Rehabilitation Assessment of the Luiz I Bridge
}

\author{
Bruno J. Afonso Costa"; Filipe Magalhães ${ }^{2}$; Álvaro Cunha ${ }^{3}$; and Joaquim Figueiras ${ }^{4}$
}

\begin{abstract}
Dynamic testing in the condition assessment of existing bridges to support their rehabilitation and strengthening designs is becoming a common procedure, yet additional dynamic testing after rehabilitation is rare. Nevertheless, combining the results of dynamic testing before and after bridge rehabilitation produces unique and valuable information regarding changes occurred in a bridge's dynamic properties. Such dual testing would allow researchers to verify the behavior predicted at the design stage and to assess the stiffness variation, which would eliminate the need for mandatory field static testing. This article reports the modal analysis of a unique centenary steel-arch bridge that was recently rehabilitated and strengthened. The ambient vibration test conducted after the construction works is described, and the data are compared with those collected before the rehabilitation. Structural identification is completed by means of experimentally validated threedimensional (3D) finite-element (FE) models simulating both phases. The results bring to light important findings concerning variation in bridge stiffness, changes in the modal parameters, and the impact of support conditions and structure of decks in bridge dynamic properties. DOI: 10.1061/(ASCE)BE.1943-5592.0000632. () 2014 American Society of Civil Engineers.
\end{abstract}

Author keywords: Centenary steel-arch bridge; Rehabilitation assessment; Modal analysis; Ambient vibration test; Three-dimensional numerical model; Structural identification.

\section{Introduction}

\section{Dynamic Testing in the Assessment of Old Steel Bridges}

Although current infrastructure maintenance and management systems, such as those related to bridges, continue to use periodic visual inspections as the main source of information, the view that this approach cannot effectively meet the growing demands of modern societies is consensual among the technical and scientific communities. In fact, if, on the one hand, the full operational potential of the structures cannot be explored by conservative decision-making processes based on scarce and nonmeasurable information, then, on the other hand, the financial resources available for repair, rehabilitation, strengthening, upgrade, or replacement are increasingly limited. Furthermore, often problems not perceived by visual inspection can only be disclosed through procedures that include the measurement of key parameters. Consequently, the use of nondestructive testing

${ }^{1} \mathrm{Ph} . \mathrm{D}$. Researcher, Faculty of Engineering, Dept. of Civil Engineering, Univ. of Porto, Rua Dr. Roberto Frias, 4200-465 Porto, Portugal (corresponding author). E-mail: bjacosta.feup@gmail.com

${ }^{2}$ Assistant Professor, Faculty of Engineering, Dept. of Civil Engineering, Univ. of Porto, Rua Dr. Roberto Frias, 4200-465 Porto, Portugal. E-mail: filipema@fe.up.pt

${ }^{3}$ Full Professor, Faculty of Engineering, Dept. of Civil Engineering, Univ. of Porto, Rua Dr. Roberto Frias, 4200-465 Porto, Portugal. E-mail: acunha@fe.up.pt

${ }^{4}$ Full Professor, Faculty of Engineering, Dept. of Civil Engineering, Univ. of Porto, Rua Dr. Roberto Frias, 4200-465 Porto, Portugal. E-mail: jafig@fe.up.pt

Note. This manuscript was submitted on November 18, 2013; approved on March 28, 2014; published online on April 30, 2014. Discussion period open until September 30, 2014; separate discussions must be submitted for individual papers. This paper is part of the Journal of Bridge Engineering, (c) ASCE, ISSN 1084-0702/05014006(11)/\$25.00. tools is emerging as a valuable solution to assist in the condition assessment of existing structures, both in terms of their load-carrying capacity and serviceability (DeWolf et al. 2002). Moreover, data obtained through these means are essential for the quantification of parameters and identification of mechanisms that are to be integrated in the numerical models that support reliable and objective structural evaluation (Farhey 2005). In this context, model calibration based on experimental measurement emerges as a key task, for which the selection of the model space and strategy for the numerical modeling and physical completeness are the kernel problems (Aktan et al. 1998).

Although some researchers have pointed out the intrinsic difficulties in performing vibration testing in large bridges, such as nonstationary excitation (Catbas et al. 2007), nonlinear boundary or continuity conditions, and nonideal connections/interfaces between structural members and components (Farhey 2005), it is unquestionable that dynamic testing stands as an innovative way of great potential in the structural identification of bridges for condition assessment (Aktan et al. 1997; DeWolf et al. 2002). It becomes imperative when assessing the seismic vulnerability of a structure and corresponding retrofit design (Cheung et al. 2007), and can play a decisive role in the rehabilitation of centenary steel bridges, both in supporting the project design and validating their performance for the new structural condition.

Several examples of dynamic tests performed in old steel bridges have been reported in technical literature, of which the most significant cases regarding repair, rehabilitation, upgrade, or strengthening projects are presented shortly herein. Ren et al. (2004b) have studied the dynamic properties of a steel girder-tied arch bridge by modal analysis and ambient vibration testing. The mode shapes and associated natural frequencies of the structure were extracted from the field data and subsequently used to validate and/or calibrate three-dimensional (3D) finite-element (FE) models. The results assisted the seismic evaluation of the bridge and its likely retrofit. Similar studies were conducted by Ye et al. (2005) on the Brooklyn Bridge and Harik et al. (1997) on a double-deck cantilever throughtruss bridge. Ermopoulos and Spyrakos (2006) and Spyrakos et al. (2004) have tested historic railway steel truss bridges still in service, 
which are part of the southern railway network of Greece. The first natural frequencies were extracted from the accelerations measured during the free-vibration response after crossing the engine tracks and then used in the calibration of the 3D FE models by comparing them with the numerical estimates. Based on these models, the loadcarrying capacity of the bridges to withstand loads specified by current codes was appraised, and the corresponding strengthening schemes were designed. Roeder et al. (2000) analyzed the safety of the Toutle River Bridge, whereas Ren et al. (2004a) assessed the Roebling Bridge. Costa et al. (2013) and Gonçalves et al. (2008) have reported the execution of dynamic tests before and after the rehabilitation of centenary steel truss bridges, and Zaki and Abu-Hamd (2007) have used dynamic testing techniques to evaluate the evolution of the modal properties of an old steel railway bridge during strengthening works carried out with no traffic disruptions. The results have proven the effectiveness of the intervention strategies.

\section{Luiz I Bridge and Its Rehabilitation}

The Luiz I Bridge constitutes a unique example of bridge engineering, known worldwide, which marked a time of the Portuguese industrial period [Fig. 1(a)]. Its distinctive feature is the existence of two decks at different levels supported by a single parabolic doublehinged arch. The 391.25-m-long upper deck (UD) is formed by two 5-m high-truss girders comprising 13 spans that rest on five metallic piers and two masonry piers (north side). The two lattice girders of the LD (LD) are suspended from the arch by four tie trusses spaced at $36-\mathrm{m}$ intervals, crossing a total span length of $174 \mathrm{~m}$. The loads applied on both decks are transferred to the abutments and piers through a roller. In general, bars pertaining to the original structure have I-, T-, or box-shaped sections built by assembling several plates and angles through riveted connections, which was a typical technique of steel construction of that time.

Despite some operations of rehabilitation and maintenance or minor changes suffered to accommodate the passage of new types of vehicles, the structure has been in continuous operation since its completion in October 1886. However, lately, a strengthening and rehabilitation process took place on this bridge to allow for the integration of its UD in the infrastructure of the Porto Metro Light Rail Network. One of the major operations fulfilled during this process was the complete replacement of the UD bridge floor system (crossbeams, stringers, and lightweight concrete pavement) by a suitable metallic profile grid capable of properly transmitting the new railway traffic loads to the truss girders [Figs. 1(b and c)]. The UD girders, suspension ties, arch diagonals, and bracing elements all over the bridge were strengthened with the addition of steel profiles. All of the original roller bearings were cleaned and lubricated, except for the supports at the UD abutments, which were replaced by disk bearings due to their severely damaged condition.

\section{Scope and Objectives}

The rehabilitation and strengthening of the bridge was carried out following an assent given by a viability study performed to analyze the structural effects that would result from using the UD to support a new light metro double line (Coelho et al. 1996). In this study, a dynamic analysis was conducted through a complete modal identification of the structure based on the execution of an ambient vibration test and the development of two-dimensional (2D) and 3D FE models, which were used to simulate the bridge response under the new service conditions (Calçada et al. 2002). The data produced by this viability study played a decisive role in the rehabilitation design by enabling the optimization of the strengthening schemes to be implemented.

Although it is not a common practice in this type of project, given the depth and complexity of the intervention undertaken on the UD, a second ambient vibration test was executed after the completion of the construction works. The collected data have allowed for the obtainment of unique and valuable information regarding the changes produced in the dynamic properties of the bridge as well as verification of the behavior predicted at the design stage. Furthermore, the stiffness variation of the UD between the two conditions was also made possible both in the transverse and vertical directions, thus making the need of a static field test not mandatory.

This article presents a study regarding the modal identification of the Luiz I Bridge, which has undergone a rehabilitation and strengthening process to enable its use for the passage of new light metro vehicles. The experimental program adopted in the ambient vibration test performed for the new service conditions as well as the data processing implemented for the extraction of the modal parameters are described, always with reference to the previous test conducted to support the viability study of the rehabilitation project. The 3D FE models that were developed to simulate the dynamic response of the bridge before and after the construction works are

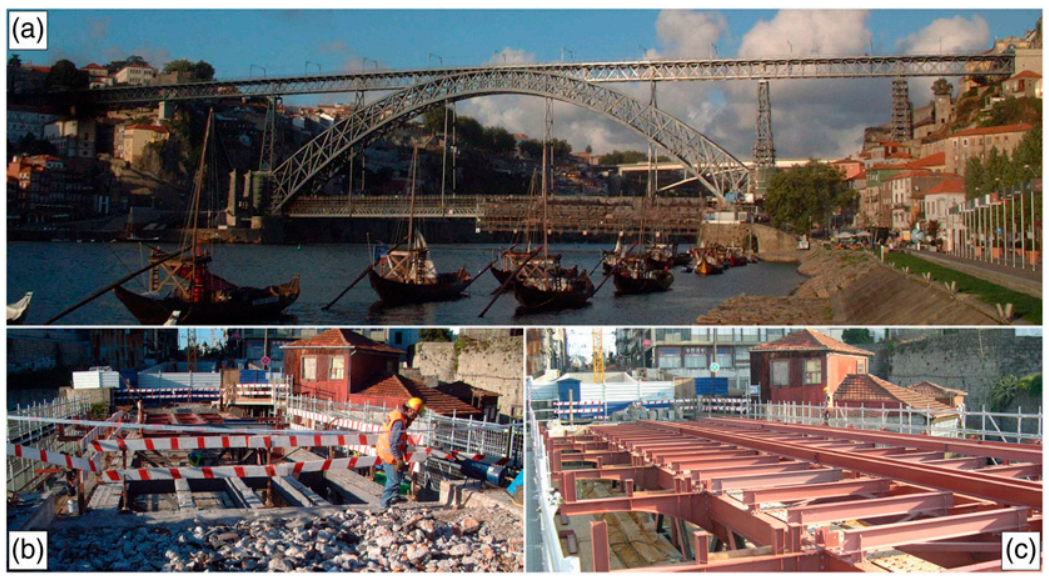

Fig. 1. Luiz I bridge: (a) general view; (b) demolition of concrete pavement and removal of old supporting steel grid (floor beams and stringers) of UD; (c) installation of new steel grid and floor system in UD (images by Bruno José Afonso Costa) 
reported, and the adopted modeling strategy is detailed. Major conclusions regarding the evolution of the level of vibration, modal parameters, and stiffness of the bridge are drawn from the direct comparison of the two sets of field results and by correlating them with the numerical estimates. Additionally, the most significant variables that control the modal parameters are also disclosed, which consequently can assist in the integration of the newly updated service model within a structural health monitoring system to identify any alterations produced over time.

\section{Ambient Vibration Testing}

The ambient vibration tests conducted before and after rehabilitation and strengthening of the bridge sought to evaluate its vibration response under normal operation, mostly induced by the wind and traffic, and to identify the modal parameters for both conditions. The first test, henceforth termed Test 1, was performed to support the viability study and subsequently the rehabilitation design. With respect to the second test, hereafter referred to as Test 2 , the dynamic measurements served to detect the changes produced in the structural behavior as a result of the construction works and establish a sound baseline for the dynamic characteristics of the bridge in the new service phase to enable its structural health monitoring. Taking into account that the description of Test 1 has already been done in a previous paper (Calçada et al. 2002), in this article, the focus is placed on the experimental procedure, data processing, and modal identification implemented in Test 2. Yet for the sake of clarity, all differences between the two tests are pointed out.

\section{Testing Program}

For recording the bridge vibrations, a total of 28 measurement sections were defined, 19 in the UD and nine in the LD, as depicted in Fig. 2. However, in Test 1, the accelerations of the LD were only collected at the joints in correspondence with the suspension ties, and during Test 2, no measurements were taken at Section 19. All sections of the UD were instrumented with two seismographs, one positioned at each side of the deck on the sidewalk limits upstream and downstream. On the LD, a single device was used to record the upstream accelerations, even though for Test 2, the vibration response has also been occasionally acquired from the downstream side to unveil the eventual torsion modes.

For the entire duration of the tests, two reference stations were set on the UD at Section 15. For Test 2, an additional reference was introduced at Section 23 (upstream). Although two recorders were permanently kept at the UD reference stations, the third reference recorder was only used during the ambient vibration test of the LD. The remaining recorders were consecutively placed at the other sections and acted as moving sensors. In all of the measuring points, the accelerations were measured along three orthogonal directions oriented according to the natural reference axes of the bridge (longitudinal, vertical, and transverse).

All four measuring devices are essentially constituted by one triaxial force balance accelerometer, an analog-to-digital (A/D) converter, a battery that enables 1-day test autonomy, and a memory card for recording the acquired data. In Test 1 , the resolution of the converters was 16 bits. For Test 2 , the resolution was enhanced to 18 bits. Furthermore, in the second test, the seismographs were upgraded with global positioning system (GPS) sensors to continuously update the time of the internal clocks; consequently, synchronization between the units was improved.

Because such units are autonomous and the definition of the acquisition timetable was previously performed by connecting each measuring unit to a laptop, the need for using long electrical cables was overcome, which permitted a faster execution of the tests. Acceleration time series were collected during setups of 6 and 16 min, with sampling frequencies of 50 and $100 \mathrm{~Hz}$ in the first and second tests, respectively. Despite these values being imposed by the filters of the acquisition equipments, the frequency content of interest for this bridge, less than $10 \mathrm{~Hz}$, was perfectly captured.

\section{Data Processing and Modal Identification}

To accurately capture the modal parameters of the bridge, particularly those associated with the vibration modes of different types with very close natural frequencies, the collected signals of each section were precombined to obtain an enhanced time series for analysis. The new vibration signals were the half-sum of the vertical accelerations, half-difference of the vertical accelerations, and halfsum of the transverse accelerations (upstream-downstream), which enabled an improved modal extraction of the vertical, torsional, and transverse bending modes, respectively.

The identification of the resonant frequencies of the bridge for both tests was accomplished in the frequency domain using the peak-picking (PP) method (Felber 1993), which is one of the simplest techniques available for data processing of the vibration response of structures subjected to natural excitation. However, for Test 2, more sophisticated identification algorithms were used to confirm the estimates supplied by the first analysis (Cunha et al. 2006). Notwithstanding the better results obtained with these techniques, for the sake of comparison of experimental estimates from both tests, only the parameters identified with the PP method are presented herein.

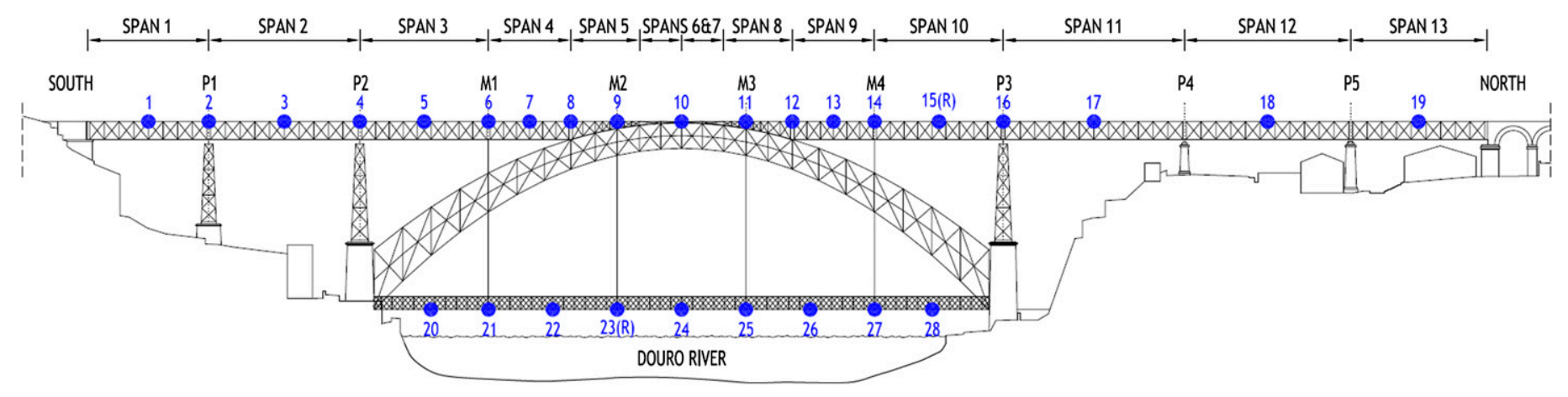

Fig. 2. Layout of measurement sections 
In light of the preceding information, normalized power spectral density (NPSD) functions were estimated from the acceleration time series of each measuring section (three precombined signals) as well as the coherence functions associated with the simultaneous measurements at the several sections. Subsequently, to highlight the natural frequencies, the average normalized power spectra densities (ANPSDs) were computed, taking into account all of the NPSDs of the same type. Although in Test 1, the 6-min records were divided into eight segments without overlapping, in Test 2, the 16-min time series were divided into time segments of 4,096 reading points (40.96 s), with an overlap of 50\%, which led to a frequency resolution of approximately $0.02 \mathrm{~Hz}$ for all spectra. Fig. 3 presents the ANPSDs of the vertical and transverse accelerations obtained for both tests in the range of $0-5 \mathrm{~Hz}$, in which the spectra of Test 2 are separated by decks.

A careful inspection of the peaks appearing in these spectra permitted the identification of the natural frequencies of the bridge. Furthermore, the plots also disclose the following:

1. Natural frequencies associated with vertical bending modes are clearly captured by the spectra obtained from the readings of both decks, thus revealing a global nature.

2. Natural frequencies associated with transverse bending modes are just local because only the movement of one of the decks is mobilized. The exception is the frequency of $0.952 \mathrm{~Hz}$, whose mode shape mobilizes the deformation of both decks [Fig. 3(b)].

The identification of the mode shapes within the range of $0-5 \mathrm{~Hz}$ was performed on the basis of the transfer functions relating the ambient response at each section with the one collected at the reference sections, which was duly validated by the corresponding coherence functions. The ratios between the values of these transfer
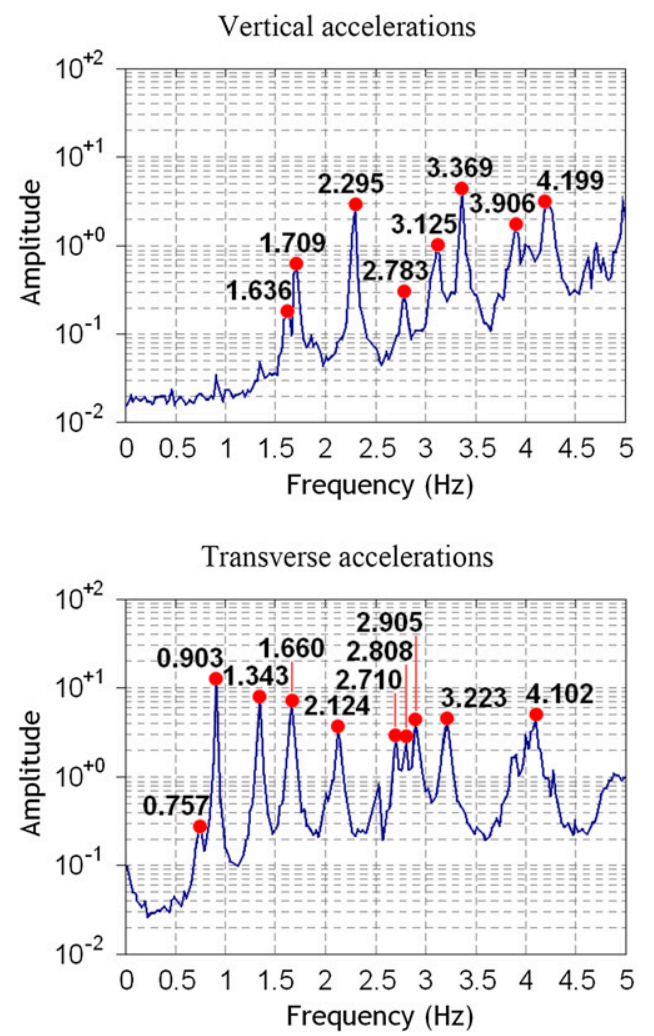

(a) functions, which were related to each natural frequency in a linear scale, enabled the estimation of the modal coordinates, whose corresponding sign was evaluated by checking the phase evolution.

In this work, no results regarding the experimental damping ratios for both bridge conditions are presented because of the high dependence of their estimation on the modal identification technique adopted. Also, for the purpose of the intended analysis, the damping values are not significant. Cunha et al. (2006) provide more information on this issue.

\section{FE Modeling}

For the purpose of this study, two complete 3D FE linear elastic models have been developed, one to simulate the bridge condition before the rehabilitation and strengthening works and the other to simulate the bridge behavior during its new service phase, henceforth referred to as Models A and B, respectively. Fig. 4 shows a general view of Model B.

\section{FES}

The models were created on the basis of two-node 3D frame elements. In some cases, four-node shell elements have also been adopted in the modeling process to simultaneously simulate the slab and plate actions of a thin 2D structural element.

Two different situations required this alternative or complementary approach. The first was related to the connection between some important elements of the structure, wherein an improvement in replicating the force transmission mechanisms was needed. This is the case of the connections of the suspension ties to the arch (upper level) and LD (lower level) and of the footings of Piers M1 and M4
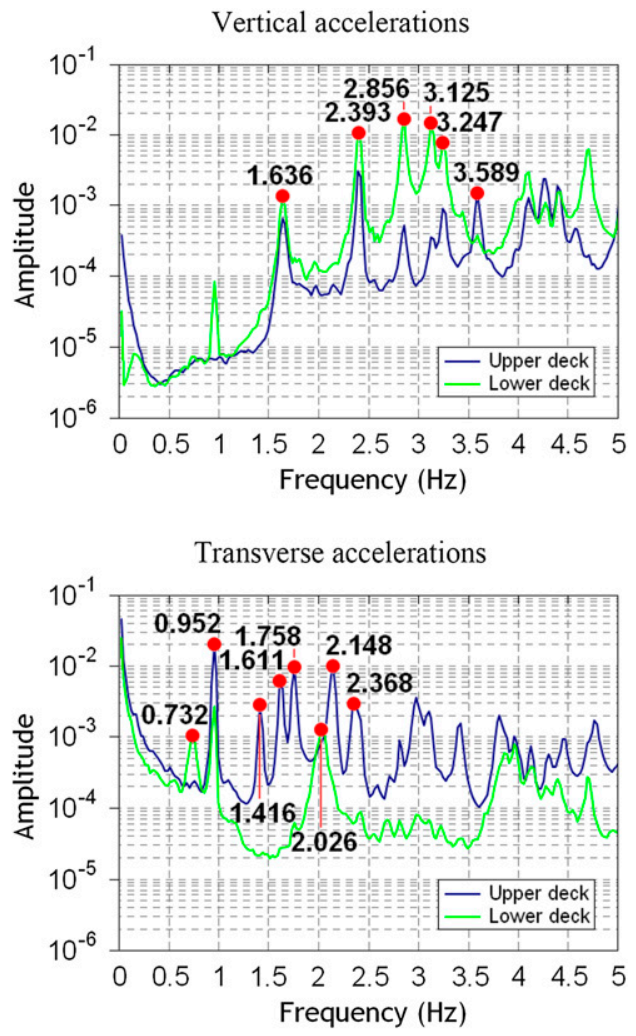

(b)

Fig. 3. ANPSDs estimated for bridge during both ambient vibration tests: (a) before rehabilitation; (b) after rehabilitation 


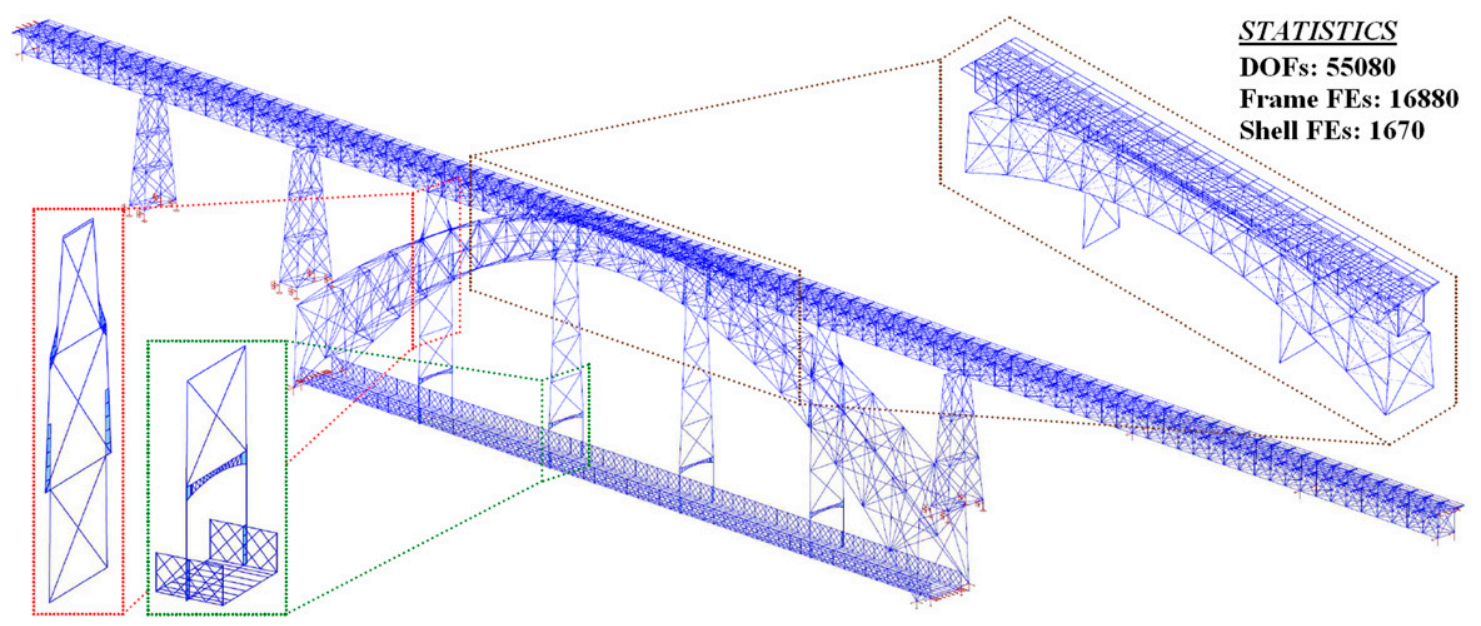

Fig. 4. FE model of Luiz I Bridge: Model B

over the arch. Fig. 4 depicts the details regarding the modeling in the alignment of the suspension ties.

The second situation was compelled by the significance that shear stresses have for the UD I-girders' response at the two spans over the arch crown. On one hand, the span-to-height ratio is low (6.4), and on the other hand, the rail loads are directly applied along these elements. For these girders, the web was modeled by means of shell elements, and the flanges were simulated with frame elements. Fig. 4 shows a detail of the UD modeling at the arch crown.

\section{Geometry, Material, Cross Section Properties, and Mass}

The geometry of the bridge and cross section properties of the elements were defined on the basis of the documents produced by the in-depth survey and inspection of the structure, which were carried out after the viability study and before the rehabilitation and strengthening works [Consultas, Estudos e Projectos de Engenharia (GRID) 2001]. The cross section properties of the diagonals, verticals, and bracings of the arch were computed according to the procedure proposed by Duan et al. (2000) to consider the actual section's integrity. For an accurate characterization of the original steel, specimens were then taken from several parts of the bridge (arch, piers, and UD) and mechanical tests were made, which included fatigue, tensile strength, and impact (Coelho et al. 1996).

With regards to the geometrical discretization of the structure, the accurate relative positioning of the elements' gravity centers was assured by adequately displacing the structural axes from the reference geometric axes. Aiming at properly capturing the stiffness contribution provided by the lightweight concrete pavement on both decks (Model A) or only on the LD (Model B), the stringers, crossbeams, and chords supporting it were modeled as steel-concrete composite bars. In the modeling of the strengthened elements of the bridge in the postrehabilitation condition, the mechanical properties were computed using equivalent elements made of a single material because the original and newly added steels do not present the same elastic parameters. Furthermore, with respect to the new steel grid on the UD that carries the loads from pedestrians and emergency road vehicles, its stiffness was taken into account by including the corresponding stringers as longitudinal bars positioned at the upper level of the deck. For the modal analysis of the models, the mass matrices were calculated assuming the whole mass was concentrated in the nodes, and all the permanent nonstructural loads were also properly converted into concentrated masses positioned at the nodes.

\section{Boundary Conditions}

The supports of both decks at the abutments only prevent the vertical and transverse displacements. However, restrictions of the longitudinal displacements of the decks had to be considered, which constituted a key issue in the FE modeling of the bridge. In the LD, the constraints are essentially caused by the steel expansion joints at both ends [Fig. 5(a)]. Three reasons decisively contribute to this fact

1. The expansion joint itself does not permit complete free movement, which is intended to favorably control the behavior of the pendulum system constituted by the LD and suspension ties.

2. At the girders' ends, the lower chord flanges laterally lean against thick steel plates attached to the abutment masonry [Fig. 5(b)].

3. The gaps that would allow some expansion movement are filled with compacted material, and during the rehabilitation process, no actions were taken to execute any cleaning.

Regarding the UD, the restraints have different causes, depending on the phase of analysis. Before rehabilitation, the extreme degradation of the steel bearings at the abutments, caused by their deficient design and lack of proper maintenance, strongly prevented the intended performance of the structure. This major inadequacy was experimentally confirmed before the beginning of rehabilitation by measuring the expansion displacements induced by the daily cyclic thermal action (Costa et al. 2004). It is also noteworthy that in this phase, the expansion joints located at the upper level were not restraining the movements because they were of a metallic cantilevered teeth type and in good condition.

After rehabilitation, the constraints are likely caused by two factors

1. At the upper level of the expansion joints, significant forces are mobilized. Indeed, steel plates covering the gap are attached to the steel structure and leaned against steel plates fixed to the masonry of the abutment. In Fig. 5(c), signs of rust in the large contact surfaces of these plates are visible.

2. Although expansion devices are applied to the rails in the vicinity of the abutments out of the UD to prevent the appearance of thermal-induced forces [Fig. 5(d)], friction is also 


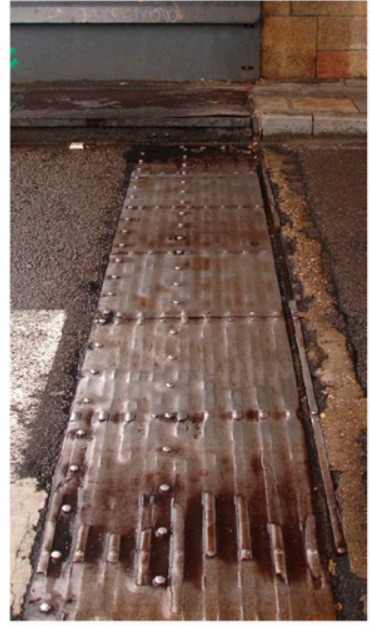

(a)

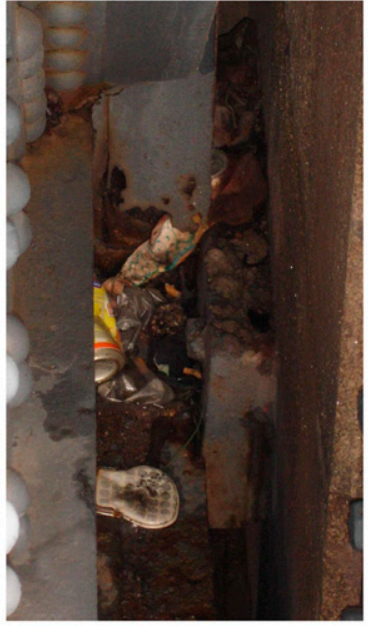

(b)

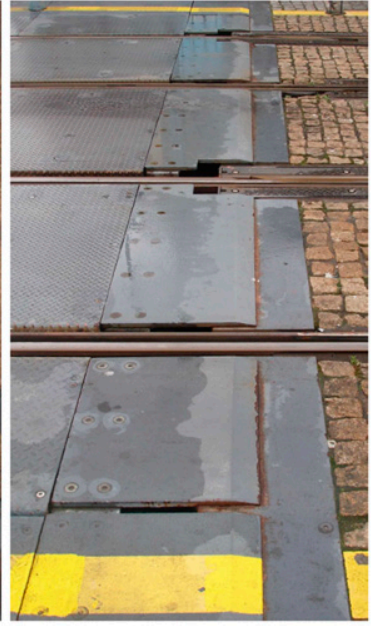

(c)

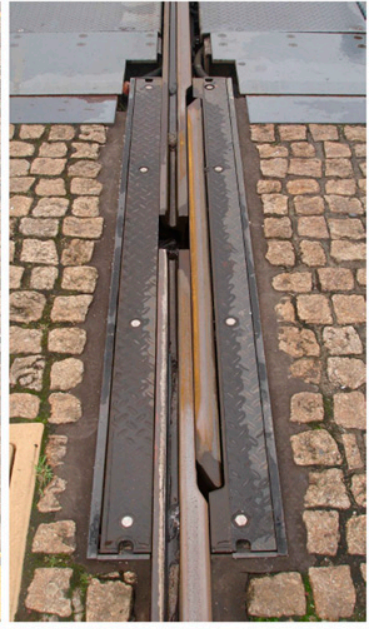

(d)

Fig. 5. Views of abutments at LD and UD: (a) expansion joint of LD; (b) contact between lower chord flanges of LD and thick steel plate attached to masonry; (c) cover plates of UD expansion joint; (d) expansion device of rail at abutment of UD (images by Bruno José Afonso Costa)

generated due to the very structure of the apparatus even though it is of a smaller magnitude.

It is also important to bear in mind that the inadequate original bearings at the abutments were replaced and the remaining UD supports were cleaned and lubricated; therefore, the constraints induced by these were minimized. In light of the preceding information, the stiffness coefficients of the springs adopted in the reference FE models to simulate the restrictions to the longitudinal displacements of both decks were estimated through a calibration procedure performed on the basis of an iterative trial-and-error process, in which the vibrating frequencies and mode shapes estimated by the numerical analysis were matched to the corresponding experimental values. The use of a model updating technique was discarded due to the noncross and localized influence of the decksupporting conditions on the vibration modes, which constituted the major source of uncertainty in the modeling.

At the masonry Piers P4 and P5 (Fig. 2), the lower nodes of the girders in the support alignments have the transverse and vertical movements completely restricted. The connection between the UD and metallic piers founded in the river banks is made by an apparatus similar to that of the masonry piers. Therefore, the devices were modeled by separately defining coincident nodes in the bottom chords of the UD girders and in the top of the small rigid vertical bars fixed to the top of the piers. Using master-slave coupling for the transverse rotations and the vertical and transverse displacements of the coincident nodes, the expansion hinge effect is properly simulated. In what concerns the bearing conditions of the arch, these were replicated by pinned supports.

\section{Analysis of Results}

\section{Vibration Levels}

The peak value of the vertical accelerations acquired in Test 2 slightly exceeded $0.20 \mathrm{~g}$, which constitutes a considerable rise compared with the maximum reading recorded in Test $1(0.12 g)$ (Calçada et al. 2002). However, this increase of the vertical vibration level does not imply a decay of the structural behavior because the main dynamic loads are quite different because the UD previously served road traffic and is now crossed by rail vehicles. Moreover, it depends on the acquisition frequency adopted in the tests, which was much higher for Test 2. On the other hand, although the new maximum vertical acceleration is a value relatively high in terms of pedestrian comfort, its relevance is mitigated by the fact that it includes significant high-frequency contributions. Similarly to the vibration response observed before the rehabilitation process, the transverse accelerations in Test 2 are also considerably lower than the verticals in a proportion of approximately 1:4.

\section{Natural Frequencies}

In this section, an analysis of the natural frequencies identified in both tests within the range of $0-3.6 \mathrm{~Hz}$ is performed. Cunha et al. (2006) analyzed the evolution of the natural frequencies of the identified modes along the setups performed in the second test and observed that during the $6 \mathrm{~h}$ of testing, the temperature varied by approximately $10^{\circ} \mathrm{C}$, and the average standard deviation of the natural frequencies was $0.62 \%$ of their mean values. A combined inspection of the power spectra presented in Fig. 3 and the experimental natural frequencies listed in Table 1 (third and sixth columns) leads to the following comments:

1. Although 15 natural frequencies were obtained in Test 1 , the quality of the field data only allowed for the reliable identification of nine vibration modes.

2. For each of the 14 natural frequencies identified in Test 2, it was possible to identify the corresponding mode shape.

3. The first vertical bending mode identified in Test 1 is associated with two natural frequencies in the corresponding ANPSD (1.636 and $1.709 \mathrm{~Hz}$ ), which suggests a likely nonstationary behavior of the structure caused by the support conditions. This specific mode shape holds a large translation component of the UD, which will be described in the following section.

Comparing the natural frequencies extracted from both tests for the same vibration modes, small differences are found that did not reach $6.6 \%$, except for the sixth mode, in which the reduction exceeded $17 \%$. Nevertheless, a slight increase of these parameters appears to be the tendency. A switch of the relative position of the fourth and fifth modes is also detected, potentiated by the close proximity of the values. 
Table 1. Summary of Natural Frequencies of the Bridge

\begin{tabular}{|c|c|c|c|c|c|c|c|c|}
\hline \multirow[b]{2}{*}{ Mode number } & \multirow[b]{2}{*}{ Mode type } & \multicolumn{3}{|c|}{ Before rehabilitation } & \multicolumn{3}{|c|}{ After rehabilitation } & \multirow[b]{2}{*}{$\Delta_{b}(\%)$} \\
\hline & & Identified $^{\mathrm{a}}(\mathrm{Hz})$ & Numerical $^{\mathrm{b}, \mathrm{c}}(\mathrm{Hz})$ & $\Delta_{a}(\%)$ & Identified $^{\mathrm{d}}(\mathrm{Hz})$ & Numerical $^{\mathrm{e}}(\mathrm{Hz})$ & $\Delta_{a}(\%)$ & \\
\hline First & First $\mathrm{T}$ & 0.757 & $0.759 / 0.821$ & $0.26 / 8.45$ & 0.732 & 0.738 & 0.82 & -3.30 \\
\hline Second & Second $\mathrm{T}$ & 0.903 & $0.908 / 0.945$ & $0.55 / 4.65$ & 0.952 & 0.925 & -2.84 & 5.43 \\
\hline Third & Third T & 1.343 & $1.400 / 1.455$ & $4.24 / 8.34$ & 1.416 & 1.399 & -1.20 & 5.44 \\
\hline Fourth & Fourth T & 1.660 & $1.657 / 1.528$ & $-0.18 /-7.95$ & 1.611 & 1.650 & 2.42 & -2.95 \\
\hline Fifth & First V-L & 1.636 & $1.636 / 1.561$ & $0.00 / 4.58$ & 1.636 & 1.662 & 1.59 & 0.00 \\
\hline Sixth & Fifth T & 2.124 & $2.016 / 1.870$ & $-5.08 /-11.96$ & 1.758 & 1.737 & -1.19 & -17.23 \\
\hline Seventh & Sixth T & - & $2.042 /-$ & - & 2.026 & 2.015 & -0.54 & - \\
\hline Eighth & Seventh T & - & $2.626 /-$ & - & 2.148 & 2.131 & -0.79 & - \\
\hline Ninth & Eighth T & - & $3.227 /-$ & - & 2.368 & 2.404 & 1.52 & - \\
\hline 10th & Second V & 2.295 & $2.274 / 2.328$ & $-0.92 / 1.44$ & 2.393 & 2.365 & -1.17 & 4.27 \\
\hline 11th & Third V & - & $2.806 /-$ & - & 2.856 & 2.801 & -1.93 & - \\
\hline 12 th & Fourth V & - & $2.949 /-$ & - & 3.125 & 2.998 & -4.06 & - \\
\hline 13th & Fifth V & 3.125 & $3.126 / 3.080$ & $0.03 /-1.44$ & 3.247 & 3.198 & -1.51 & 3.90 \\
\hline 14 th & Sixth V & 3.369 & $3.399 / 3.469$ & $0.89 / 2.97$ & 3.589 & 3.362 & -6.32 & 6.53 \\
\hline
\end{tabular}

Note: Vibration modes are numbered according to the order of the identified modes after rehabilitation. $\mathrm{L}=$ longitudinal mode; $\mathrm{T}=$ transverse mode; $\mathrm{V}$ $=$ vertical mode; $\Delta_{a}=$ numerical/identified $-1 ; \Delta_{b}=$ identified after rehabilitation/identified before rehabilitation -1 .

${ }^{\mathrm{a}}$ Natural frequencies identified before the rehabilitation.

${ }^{\mathrm{b}}$ Natural frequencies estimated with Model A.

${ }^{\mathrm{c}}$ Natural frequencies estimated with the model developed for the viability study.

${ }^{\mathrm{d}}$ Natural frequencies identified after the rehabilitation.

${ }^{\mathrm{e}}$ Natural frequencies estimated with Model B.

One peculiar aspect that is highlighted from the identification of the natural frequencies is the fact that the value of the first vertical bending mode (fifth mode) remained unaltered, despite the significant operations performed on the UD. From a structural point of view, this fact is a fortunate coincidence because it was not specifically planned by the rehabilitation design. In addition, given the close proximity of the natural frequencies and the frequency resolution achieved for both tests of approximately $0.02 \mathrm{~Hz}$, the estimated values may fall within the same measuring interval. On the other hand, with the use of alternative identification methods, some difference could be found (Cunha et al. 2006).

Table 1 also shows the natural frequencies calculated from the numerical Models A and B (fourth and seventh columns) as well as the percentage variation from the field estimates, $\Delta_{a}$. It is noteworthy that for the analysis of the results representative of the phase before rehabilitation, two sets of numerical values are used: one regarding the numerical simulations performed within this work and the other containing the values predicted within the viability study (Calçada et al. 2002).

In general, with regards to the numerical frequencies supplied by the models of this work, the correlation with the experimental values can be classified as very good, with the average of the absolute deviations of 1.35 and $1.99 \%$, respectively, for the prerehabilitation and postrehabilitation phases. This slight increase of deviation for the second condition may be caused by a lower accuracy of Model B as a result of the existence of additional uncertainties, albeit of small influence. Consequently, the adopted modeling strategy proved to be extremely feasible in accurately replicating the natural frequencies of the bridge. Furthermore, the numerical model that simulates the dynamic behavior of the bridge before rehabilitation shows a clear improvement with respect to the one developed for the viability study, which points to an accuracy increase of four times.

Another aspect of interest in the numerical results is the changes in the ordering of the vibration modes. The close proximity of the natural frequencies of the ninth and 10th modes (eighth transverse and second vertical bending modes) after rehabilitation made their numerical calculation more difficult, resulting in the inversion of the modes. More importantly, the ordering of the vibration modes from the eighth to the 12 th position is completely altered between Models $\mathrm{A}$ and $\mathrm{B}$.

\section{Mode Shapes}

Figs. 6 and 7 show the mode shapes provided by the numerical Models A and B, which are represented in the left- and right-hand sides, respectively. The deformed shape of the bridge is defined by the upper chords of the UD and the lower chords of the LD. Even though transverse bending modes engage the torsion deformation of the UD to some extent, their configuration is represented in the figure by plan views, whereas the vertical bending modes are presented in elevation. Simultaneously, the modal coordinates extracted from both tests are plotted over the numerical mode shapes to enable an easy and immediate evaluation of the results. The analysis of the correlation between the identified and calculated parameters is accomplished by graphical comparison and by means of the modal assurance criterion (MAC) (Allemang 2003).

In general, the experimental modal components compare well with the mode shapes estimated by the models, exhibiting a slightly better match for the postrehabilitation condition, particularly with respect to the transverse modes, which is a consequence of the upgrade in the measuring devices and improvement of the testing parameters and data processing. Yet it should be pointed out that for some vibration modes, the similarity level is poor as a consequence of the lower quality of the field test data. These are the cases of the fifth transverse bending mode (sixth mode) before rehabilitation and the sixth vertical bending mode (14th mode) in the new condition.

The changes produced in the structure response regarding its stiffness can also be inferred by a careful examination of the mode shapes. The transverse bending modes after rehabilitation reveal a less smoothly deformed shape of the UD near the steel piers. Because the strengthening of these structural elements was very limited, this fact suggests a clear decrease of the transverse bending stiffness of the UD, which will be properly analyzed in the following section. 

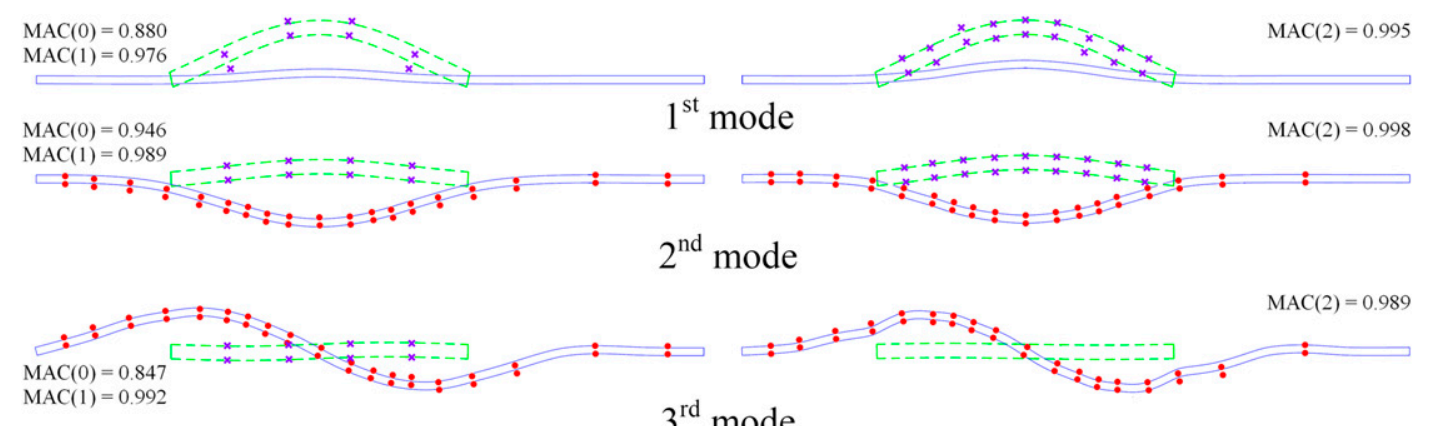

$3^{\text {rd }}$ mode
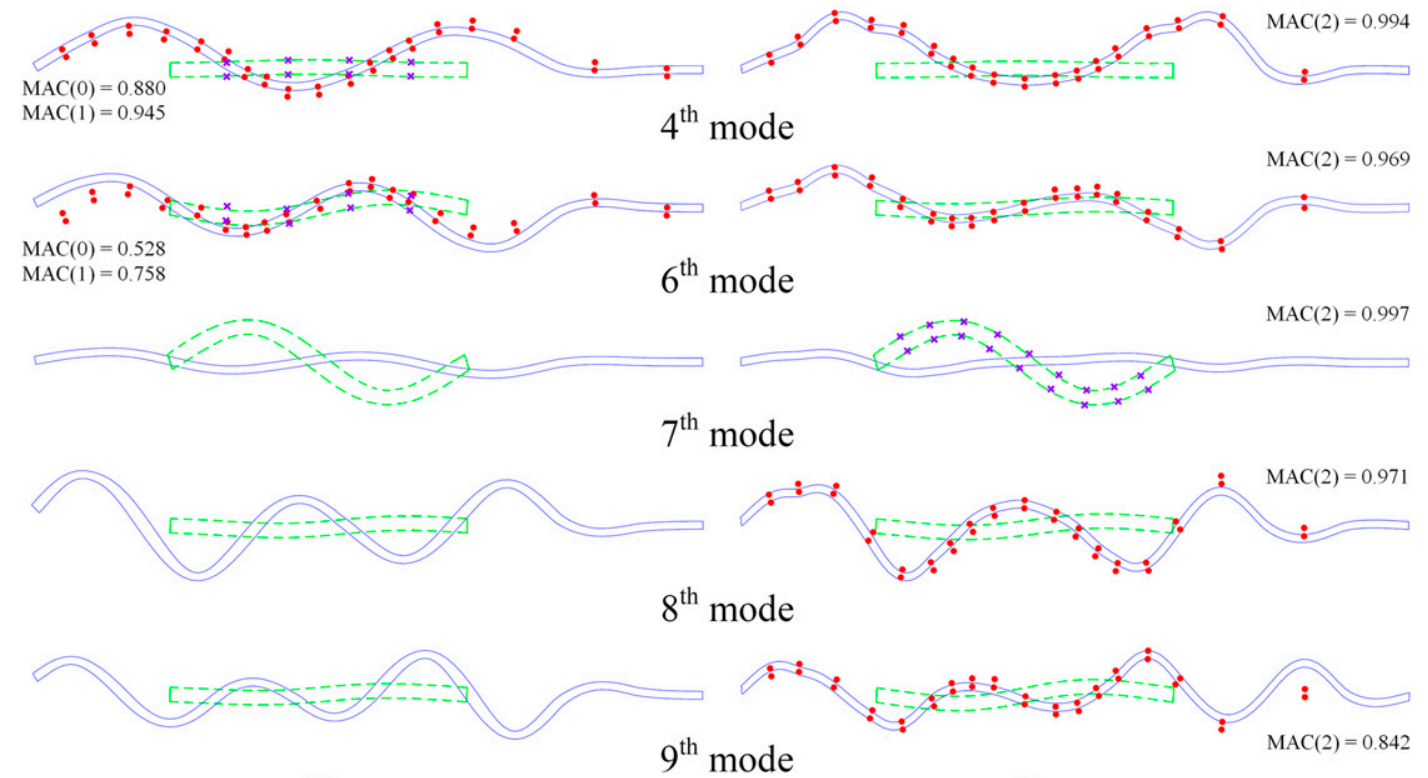

(a)

(b)

Experimental results: $\bullet$ upper deck, $\mathbf{x}$ lower deck.

Numerical results: — upper deck, ---- lower deck.

Fig. 6. Identified transverse mode shapes (a) before and (b) after rehabilitation

The MAC values comparing the numerical and experimental vibration modes for the prerehabilitation and postrehabilitation conditions, labeled $\operatorname{MAC}(1)$ and $\operatorname{MAC}(2)$, respectively, are also shown in Figs. 6 and 7 beside each mode shape. The calculation of these indicators was made on the basis of the modal displacements obtained at the measuring sections for each direction of interest: transverse or vertical. Additionally, the values estimated with the model developed for the viability study, termed $\operatorname{MAC}(0)$, are also presented.

As can be seen, the indicators $\mathrm{MAC}(1)$ and $\mathrm{MAC}(2)$ are higher than 0.90 for almost all of the vibration modes. The values of the sixth and 14th modes regarding Tests 1 and 2, respectively, confirm the lower quality of the corresponding experimental data once more. When excluding these two modes, the average value of $\mathrm{MAC}(1)$ and $\mathrm{MAC}(2)$ is 0.96 and 0.98 , respectively. However, as would be expected, a trend of degradation of these indicators as the mode order increases is perceptible, which is explained by the fact that the deformed shapes are successively more winding and therefore require a larger number of points to be captured. The clear improvement of the results calculated from Model A compared with the values from the model of the viability study is also evidenced. Therefore, in view of the preceding information, the reliability of the numerical models developed in this work for the accurate replication of the bridge modal parameters is once again highlighted.

\section{Stiffness Variation}

The change of the natural frequencies of a structure, in particular of a bridge, is an issue of extreme value because the structural performance may significantly vary depending on the characteristics of the dynamic loading. Yet the stiffness of the bridge as a whole and its relative distribution among the structural components decisively controls the static response. Therefore, if data collected from dynamic testing could be used to estimate the stiffness change from a reference state, as is the case of the prerehabilitation condition, the information would be extremely useful. Moreover, some advantages of the ambient vibration testing compared with the static load tests, such as nondisruption of the traffic and fastness of the execution, may compel the selection of the first, particularly in the context of in-service monitoring.

The evaluation of the stiffness variation based on modal data requires a judicious selection of the vibration modes but also an accurate quantification of the mobilized mass. The expression for the modal calculation of this parameter, $\Delta K$ (modal stiffness), between the two structural conditions is given by the following equation:

$$
\Delta K=(\Delta f)^{2} \times \Delta M
$$

where $\Delta f$ and $\Delta M=$ proportional variations of the natural frequency and modal mass, respectively, computed as a postrehabilitation to 


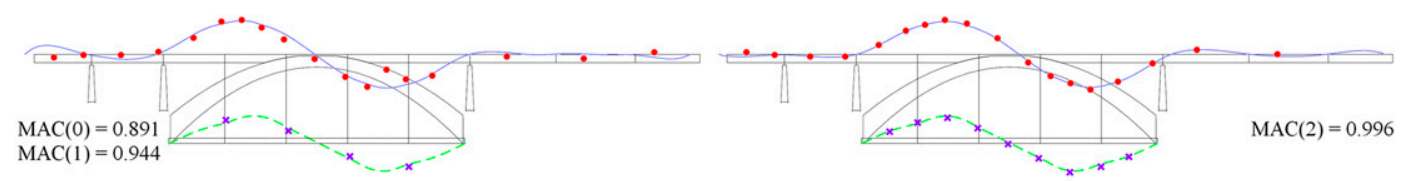

$5^{\text {th }}$ mode

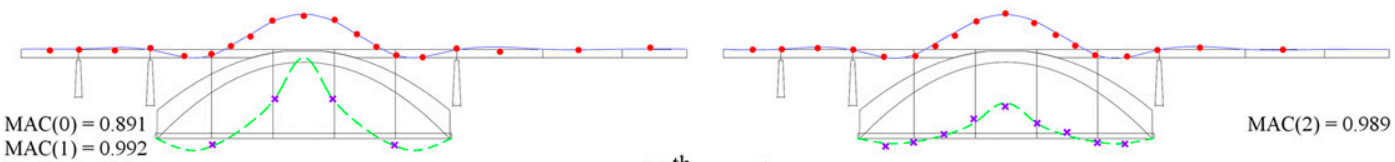

$10^{\text {th }}$ mode

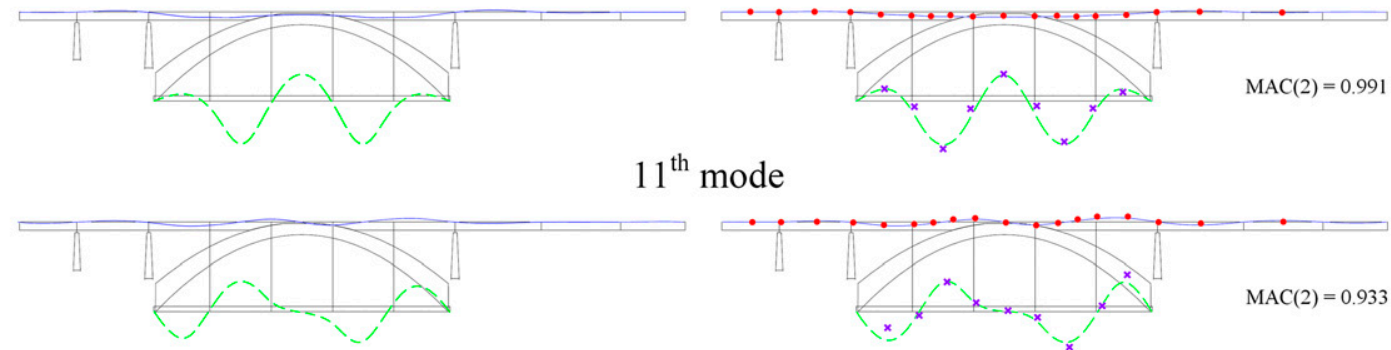

$12^{\text {th }}$ mode

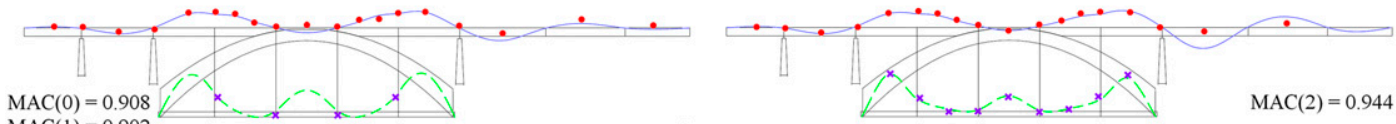

$13^{\text {th }}$ mode

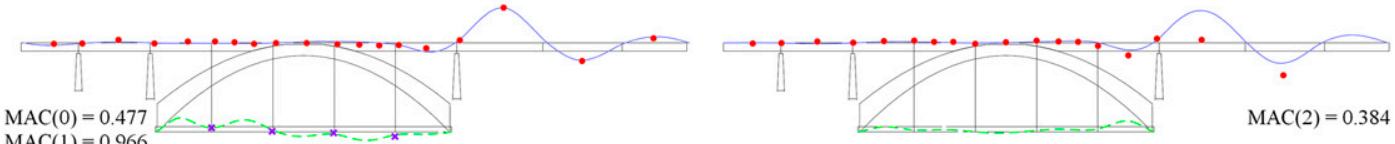

(a)

$14^{\text {th }}$ mode

(b)

Experimental results: $\bullet$ upper deck, $\times$ lower deck.

Numerical results: — upper deck, ---- lower deck.

Fig. 7. Identified vertical mode shapes (a) before and (b) after rehabilitation

prerehabilitation condition ratio. To use this equation, Table 1 gives the percentage deviations of the natural frequencies identified in both tests, and the modal masses for each direction of interest were estimated from the numerical analyses.

To appraise the variation of the vertical bending stiffness experienced by the UD, the vertical vibration mode to be selected must have a configuration for which the deformation shape of the remaining elements is minimal. The most suitable candidate is therefore the sixth vertical vibration mode (14th mode) where the deformation is almost restricted to the four north spans (Fig. 7). Table 2 lists the relevant quantities from which a stiffness reduction of $18 \%$ is estimated, which is a value very close to that inferred from the static load tests carried out on the bridge (Costa 2013).

With regard to the change of the transverse bending stiffness of the UD, the task is made more difficult due to the fact that in all transverse modes, the arch and piers are deformed. The selection of the candidate vibration mode met the following criteria:

1. Nonexistence of rotation at the arch crown;

2. Minimization of the transverse deformation of the arch and metallic piers; and

3. Existence of at least one UD span whose deformation has inflection points and small transverse displacements at the supports.
The most suited vibration mode is the seventh transverse (eighth mode) and the best fitting span is the 11th (Figs. 2 and 6). Because this mode shape was not identified in Test 1 , the data used for the analysis are supplied by the numerical analysis. Nevertheless, the validity of the conclusions is ensured by the reliability of the models developed for this work. Based on the data listed in Table 2, the reduction of the transverse bending stiffness of the UD is estimated at $58 \%$. A static analysis of Span 11, considered as a single simply supported bridge, points to a close value of stiffness decrease (54\%) when a uniform transverse load is applied, thus proving the adequacy of the adopted procedure.

\section{Key Parameters in Bridge Dynamics}

The contribution of several structural parameters for the accurate definition of the modal parameters of the bridge was also studied by sequentially changing their quantities in parallel numerical analyses. The variables under analysis were the

1. Restriction level of the decks' longitudinal displacements;

2. Effect of the lightweight concrete pavements;

3. Contribution of the UD stringers in the new bridge condition; and 
Table 2. Stiffness Variation of the UD

\begin{tabular}{lccccc}
\hline Direction & $\Delta f$ & $\Delta M$ & $\Delta K_{1}$ & $\Delta K_{2}$ & Deviation $(\%)$ \\
\hline Vertical & $3.589 / 3.369=1.065$ & $144.0 / 199.1=0.723$ & 0.821 & 0.833 & -1.46 \\
Transverse & $2.131 / 2.626=0.812$ & $470.9 / 736.8=0.639$ & 0.421 & 0.456 & -7.70 \\
\hline
\end{tabular}

Note: $\Delta f=$ proportional variation of the natural frequency; $\Delta M=$ proportional variation of the mass; $\Delta K_{1}=$ stiffness variation estimated through Eq. (1); $\Delta K_{2}$ $=$ stiffness variation estimated from static testing or analysis.

4. Transverse bending stiffness of the UD at the upper level. The collected results lead to the following comments:

- Constraints on the longitudinal displacements of the deck ends only influence a limited number of vibration modes: the two transverse modes where the deformed shape of the LD is predominant (local modes) and the first vertical mode to which a significant component of longitudinal movement on the UD is associated (Fig. 7).

- There is no cross effect of the support conditions at both deck ends on the vibration modes influenced by them, e.g., restrictions to the longitudinal displacements of the UD do not generate changes in the natural frequencies of the vibration modes associated with the movement of the LD. This fact has enabled the calibration and/or update of the elastic coefficients of the longitudinal springs on the basis of a small number of vibration modes (first, fifth, and seventh modes) by matching the numerical frequencies with the field-measured values.

- The lightweight concrete pavement caused a general stiffening of the bridge in its prerehabilitation condition. The influence on the transverse modes associated with a large deformation of the LD was very strong, but the impact on the remaining transverse modes was also very significant. For the new condition of the bridge, the influence of the concrete pavement stiffness is substantially smaller, yet it influences the transverse local modes of the LD to the same extent and the vertical vibration modes only slightly.

- With respect to the UD and for the new service condition, both of the stringers of the new steel grid (main) and new floor system (secondary) have a minor influence on the global transverse modes and also affect the first vertical mode, which holds a large longitudinal movement of the UD.

\section{Summary and Conclusions}

This article has presented a study regarding the modal identification of a centenary double-deck steel-arch bridge, which underwent rehabilitation and strengthening work to integrate its UD into the light metro infrastructure network of Porto. Two ambient vibrations tests were conducted to collect data from the prerehabilitation and postrehabilitation conditions. Data from the first test were used to assist the viability study of the project and subsequently its design, whereas the measurements from the second test, detailed herein, helped to identify the changes produced in the behavior of the bridge for the new service conditions and also provided a sound baseline for structural health monitoring. The design and execution of the second test were comprehensively reported, which included the testing procedures, instrumentation, and signal acquisition, and differences from the first test were pointed out. Additionally, the data processing and modal extraction techniques implemented for the structural identification were also presented. The 3D FE models were constructed to support the modal analysis, and in turn, experimental data served to validate and/or update the numerical models. Besides simulating the prerehabilitation and postrehabilitation conditions of the bridge, the two new base models have enabled the assessment of the influence of several structural parameters on the dynamic properties.

The results of this study led to the following conclusions:

1. The measured natural frequencies for the same vibration modes have experienced small changes, presenting a slight tendency to increase after bridge rehabilitation.

2. With respect to the mode shapes, the deformed configuration of the UD became less smooth near the steel piers for the transverse vibration modes, particularly as the order increased.

3. The modal parameters identified from both tests corresponded very well with the estimates supplied by the numerical models developed for this study.

4. The new model that replicates the prerehabilitation condition of the bridge has provided estimates of improved quality in relation to those that had been calculated in the viability study.

5. The variation of the frequencies indicates that the vertical bending stiffness of the UD was reduced by $18 \%$, whereas the transverse one reached a decrease of $58 \%$. The values were very close to the static estimates, either experimental or numerical.

6. Constraints on the longitudinal displacements of both decks decisively contribute to control of the natural frequencies of specific vibration modes, which has allowed a simple and reliable calibration procedure to quantify the stiffness constants adopted in the longitudinal springs of the model supports.

7. The lightweight concrete pavements of the decks clearly stiffen the bridge, and the new floor system and steel grid of the UD have a comparatively smaller impact on the natural frequencies.

It is worth mentioning that some issues raised in this work require further efforts to confirm some adopted procedures or evaluate the significance of some experimental findings. In the near future, advanced optimization techniques for modal updating should be used to verify or improve the adopted numerical models, and continuous periodic dynamic monitoring of the bridge should be accomplished to assess the real impact of temperature on its modal parameters.

\section{Acknowledgments}

The authors gratefully acknowledge the financial support provided by the Portuguese Scientific Foundation (FCT-MCES) to the first two authors through grants with the references SFRH/BD/13138/ 2003 and SFRH/BD/24423/2005, respectively. The authors also thank the bridge owner Metro do Porto, S.A., for their assistance and cooperation. Finally, the authors are grateful for the information provided by the Instituto da Construção (FEUP), responsible for the viability study, and by the designer of the rehabilitation project GRID-Consultas, Estudos e Projectos de Engenharia.

\section{References}

Aktan, A. E., et al. (1997). "Structural identification for condition assessment: Experimental arts." J. Struct. Eng., 10.1061/(ASCE)0733-9445 (1997)123:12(1674), 1674-1684. 
Aktan, E., Çatbaş, N., Türer, A., and Zhang, Z. (1998). "Structural identification: Analytical aspects." J. Struct. Eng., 10.1061/(ASCE)07339445(1998)124:7(817), 817-829.

Allemang, R. (2003). "The modal assurance criterion-Twenty years of use and abuse." J. Sound Vibrat., 37(8), 14-21.

Calçada, R., Cunha, A., and Delgado, R. (2002). "Dynamic analysis of metallic arch railway bridge." J. Bridge Eng., 10.1061/(ASCE)10840702(2002)7:4(214), 214-222.

Catbas, F. N., Ciloglu, S. K., Hasancebi, O., Grimmelsman, K., and Aktan, A. E. (2007). "Limitations in structural identification of large constructed structures." J. Struct. Eng., 10.1061/(ASCE)0733-9445(2007) 133:8(1051), 1051-1066.

Cheung, M. M. S., Noruziaan, B., and Yang, C.-Y. (2007). "Health monitoring data in assessing critical behaviour of bridges." Struct. Infrastruct. Eng., 3(4), 325-342.

Coelho, A. G., et al. (1996). "Viability study for the integration of Luiz I Bridge upper deck in the Light Porto Metro Network." Technical Rep., Instituto da Construção, Faculty of Engineering, Univ. of Porto, Porto, Portugal (in Portuguese).

Consultas, Estudos e Projectos de Engenharia (GRID). (2001). "Geometry survey and inspection of Luiz I Bridge: Performed works and tests." Technical Rep., Lisbon, Portugal.

Costa, B. J. A. (2013). "Structural identification of old steel bridges: Monitoring and rehabilitation assessment." Ph.D. thesis, Faculty of Engineering, Univ. of Porto, Porto, Portugal.

Costa, B. J. A., Félix, C., and Figueiras, J. A. (2004). "Complementary monitoring to the present condition of the Luiz I Bridge." Technical Rep., Faculty of Engineering, Univ. of Porto, Porto, Portugal (in Portuguese).

Costa, B. J. A., Magalhães, F., Cunha, Á., and Figueiras, J. (2013). "Rehabilitation assessment of a centenary steel bridge based on modal analysis." Eng. Struct., 56, 260-272.

Cunha, A., Magalhães, F., and Caetano, E. (2006). "Output-only modal identification of Luiz I Bridge before and after rehabilitation." Proc., Int. Conf. on Noise and Vibration Engineering (ISMA2006), P. Sas and M. De Munck, eds., Dept. of Mechanical Engineering, Katholieke Univ. Leuven, Heverlee, Belgium, 1165-1178.

DeWolf, J. T., Lauzon, R. G., and Culmo, M. P. (2002). "Monitoring bridge performance." Struct. Health Monit., 1(2), 129-138.
Duan, L., Reno, M., and Lynch, J. (2000). "Section properties for latticed members of San Francisco-Oakland Bay Bridge." J. Bridge Eng., 10.1061/(ASCE)1084-0702(2000)5:2(156), 156-164.

Ermopoulos, J., and Spyrakos, C. C. (2006). "Validated analysis and strengthening of a 19th century railway bridge." Eng. Struct., 28(5), 783 792.

Farhey, D. N. (2005). "Bridge instrumentation and monitoring for structural diagnostics." Struct. Health Monit., 4(4), 301-318.

Felber, A. J. (1993). "Development of a hybrid bridge evaluation system." Ph.D. thesis, Univ. of British Columbia, Vancouver, Canada.

Gonçalves, F., Amaral, V., and Barata, D. (2008). "Rehabilitation and structural strengthening of the Fão Bridge over the Cávado River in Portugal." Proc., 7th Int. Conf. on Steel Bridges (ICSB'08), P. J. S. Cruz, L. S. da Silva, and F. Schroter, eds., European Convention for Constructional Steelwork (ECCS), Brussels, Belgium.

Harik, I. E., et al. (1997). "Free and ambient vibration of Brent-Spence Bridge.” J. Struct. Eng., 10.1061/(ASCE)0733-9445(1997)123:9(1262), $1262-1268$.

Ren, W.-X., Harik, I. E., Blandford, G. E., Lenett, M., and Baseheart, T. M. (2004a). "Roebling suspension bridge. II: Ambient testing and live-load response.” J. Bridge Eng., 10.1061/(ASCE)1084-0702(2004)9:2(119), $119-126$.

Ren, W.-X., Zhao, T., and Harik, I. E. (2004b). "Experimental and analytical modal analysis of steel arch bridge." J. Struct. Eng., 10.1061/(ASCE) 0733-9445(2004)130:7(1022), 1022-1031.

Roeder, C. W., MacRae, G., Crocker, P., Arima, K., and Wong, S. (2000). "Dynamic response and fatigue of steel tied-arch bridge." J. Bridge Eng., 10.1061/(ASCE)1084-0702(2000)5:1(14), 14-21.

Spyrakos, C. C., Raftoyiannis, I. G., and Ermopoulos, J. C. (2004). "Condition assessment and retrofit of a historic steel-truss railway bridge." J. Constr. Steel Res., 60(8), 1213-1225.

Ye, Q., Fanjiang, G.-N., and Yanev, B. (2005). "Investigation of the dynamic properties of the Brooklyn Bridge." Sensing issues in civil structural health monitoring, F. Ansari, ed., Springer, Dordrecht, Netherlands, 65-72.

Zaki, M. A., and Abu-Hamd, M. H. (2007). "Rehabilitation assessment of a steel railway bridge by dynamic field testing." Struct. Infrastruct. Eng., 3(4), 343-353. 